\title{
Durability of crosslinked polyethylene in human-contact applications: Stabilisation challenges
}

S. Al-Malaika, and C. Jefferies

Citation: AIP Conference Proceedings 1981, 020002 (2018); doi: 10.1063/1.5045864

View online: https://doi.org/10.1063/1.5045864

View Table of Contents: http://aip.scitation.org/toc/apc/1981/1

Published by the American Institute of Physics

\section{Articles you may be interested in}

Novel polyelectrolyte membranes for fuel and flow batteries: Insights from simulations

AIP Conference Proceedings 1981, 020004 (2018); 10.1063/1.5045866

Antimicrobial biodegradable coatings based on LAE for food packaging applications

AIP Conference Proceedings 1981, 020010 (2018); 10.1063/1.5045872

Tribology of innovative polybenzimidazole (PBI) coatings

AIP Conference Proceedings 1981, 020001 (2018); 10.1063/1.5045863

Preface: 9th International Conference on "Times of Polymers and Composites"

AIP Conference Proceedings 1981, 010001 (2018); 10.1063/1.5045862

Relations of pressure and temperature dependences of the Johari-Goldstein $\beta$-relaxation to the $\alpha$-relaxation: Amorphous polymers

AIP Conference Proceedings 1981, 020003 (2018); 10.1063/1.5045865

Shape memory electrospun nonwovens based on crosslinked poly( $\varepsilon$-caprolactone) for multifunctional biological applications

AIP Conference Proceedings 1981, 020006 (2018); 10.1063/1.5045868 


\title{
Durability of Crosslinked Polyethylene in Human-Contact Applications: Stabilisation Challenges
}

\author{
S. Al-Malaika*, C. Jefferies \\ Aston University \\ Polymer Processing \& Performance Research Unit \\ School of Engineering \& Applied Science, Aston Triangle \\ Birmingham B4 7ET, England, UK \\ *Corresponding author: S.Al-Malaika@aston.ac.uk
}

\begin{abstract}
Highly crosslinked polyethylene polymers (XLPE) are used in many human-contact applications that require assurance on aspects of durability, longevity and safety. The work described in this paper focuses on applications in the medical field and in the construction industry. These are related to the use of XL-UHMWPE for articulating surfaces in orthopaedic implants particularly in total hip and knee replacements, and the use of XL-HDPE for potable water pipes. Safety, performance and durability of the end-use products are mandatory for their approval and use in such applications. However, it has long been recognised that the stabilisation of such highly crosslinked polymers by commercial antioxidants (AO) faces many challenges including the possible interference of the AOs with the crosslinking step, as well as the issues of safety associated with AO leaching and migration of into the humanenvironment. Here, the effect of AOs on the long-term oxidative stability of the end-use polymer artefacts in these applications is examined and compared with that obtained with some new alternative stabilisation systems. Results show that, in both applications, it was possible to achieve superior levels of retention of the AO in the polymer and long-term thermal stabilising (LTTS) performance (ageing) with minimum interference with the crosslinking process, thereby addressing issues of stabilisation, health and safety of polymers used in direct contact with the human environment.
\end{abstract}

Keywords: crosslinked polyethylene; Long term thermal stability; UHMWPE for orthopaedic implants PACS Codes: $81.05 . \mathrm{Lg} ;$ 83.80.Sg ; 82.35.-x ; 82.35.Lr ; 82.80.GK

\section{INTRODUCTION}

Highly crosslinked polyethylene polymers, XLPE, are used in different applications including the medical and construction industries. In the medical field, particularly for hip and knee replacements, ultra-high molecular weight polyethylene, UHMWPE, is used for preparing articulating surfaces (e.g. tibia component used as a spacer between the metal components of the total knee implant). The polymer is typically crosslinked using high energy irradiation to increase the wear resistance of the finished article which is critically important for this application. In the construction industry, high density polyethylene, HDPE, is typically used for manufacturing crosslinked water-pipes for potable water in which the crosslinking process is normally carried out by using peroxides (referred to here as PEX $_{a}$ pipes) although high energy irradiation may be used too. For these human-contact applications, safety and

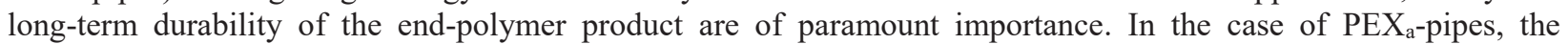
guaranteed service life is typically about 50 years. For orthopaedic implants, the polymer should ideally survive without the need for a revision surgery to replace the original polymer component. Generally, knee and hip replacements offer significant improvements to the quality of life of patients, but wear of the UHMWPE spacer over time (due to inadequate stability of the polymer) could lead to a loosening of the implant and failure, thus the need for a revision procedure which is more complex than the original surgery. The oxidative stability of the polymer in both these applications is therefore a critically important consideration and highly effective stabilisation against oxidative degradation, during both the manufacturing stage and end-use application, is crucial.

The work described here aims at addressing the challenges encountered with the use of conventional antioxidants in the commercial production of hip and knee orthopaedic implants and $\mathrm{PEX}_{\mathrm{a}}$ pipes. Some aspects of performance and durability of such crosslinked artefacts are examined and compared with those produced, in the presence of new stabilising systems, under similar commercial production conditions to assess issues of oxidative stability, permanence and longevity.

9th International Conference on "Times of Polymers and Composites"

AIP Conf. Proc. 1981, 020002-1-020002-4; https://doi.org/10.1063/1.5045864

Published by AIP Publishing. 978-0-7354-1697-0/\$30.00 


\section{STABILISATION OF XLPE AND ITS CHALLENGES}

High levels of crosslinking of PEs are critical product design consideration for the production of orthopaedic implants and potable water pipes. Peroxide-crosslinked HDPE pipes and radiation ( $\gamma$ - or e-beam) crosslinked medical-grade UHMWPE tibia components were used in this study; in both cases, high levels of crosslinking (in absence of stabilisers) were achieved, see Fig. 1. In the case of medical implants, particularly for hip and knee prosthesis, one of the main problems associated with non-crosslinked polymer is its continuous wear which gives rise to particulate wear-debris engendering osteolysis and loosening of the implant devices, thereby compromising their longevity and raising the spectre of revision surgeries to patients [1]. A revision surgery is more complicated than a primary hip or knee replacement procedure and may not provide the same lifespan of the initial replacement. However, crosslinking the UHMWPE has been shown to address this problem fairly successfully by dramatically reducing the volumetric wear of the polymer compared to conventional uncrosslinked UHMWPE [1]. Also, in the case of crosslinked HDPE for water pipes, achieving high levels of crosslinking is equally important for improving the mechanical properties and the thermal stability of the inservice pipes, particularly under load and pressure [2].

The use of high energy irradiation or peroxides for crosslinking the polymers (in the case of medical implants, the former is also used for sterilisation of the device), however, presents a major challenge. In both cases, large amounts of free radicals are generated but only some of these are used-up for the crosslinking process; the unutilised surplus of radicals remain trapped inside the polymer structure some of which would be free to attack the hydrocarbon chain thereby undermining the stability of the polymer - an intrinsic design parameter for product performance and durability - during the manufacturing stage and ultimate failure of the product in its end-use oxidative environment [3]. For example (for a peroxide-crosslinked $\mathrm{PEX}_{\mathrm{a}}$ pipe produced in the absence of antioxidants) we observe a high level of oxidation (see FTIR, Fig. 2) which is revealed by the prominent appearance of an intense carbonyl absorption envelope in the 'fingerprint' region of polymer oxidation products including ketones (peak@1720 $\mathrm{cm}^{-1}$ ); by comparison, no oxidation is seen in the case of the un-crosslinked corresponding virgin polymer. To combat the problem of oxidation, and to increase the durability and lifetime of the products, AOs are necessarily used to stabilise such highly crosslinked polymers.

Conventional thermal antioxidants, AOs, such as hindered phenols (and also hindered amines) have themselves been shown to pose specific problems in polymers used for such end-use applications [4]. The first is associated with the issue of safety due to the potential loss of the AOs through migration or leaching into the contact human-environment, see section 4 later. There is also the issue of interference of free radical scavenging antioxidants, e.g. hindered phenols, with the polymer free-radical crosslinking (XL) process, and consequent depletion of the AO-concentration. For example, in the case of radiation-crosslinked UHMWPE for hip and knee prosthesis, the natural hindered phenol antioxidant vitamin E ( $\alpha$-tocopherol, VE; Scheme 1, I), which is the only AO approved by the FDA for this application, is shown to interfere with the crosslinking process which is clearly illustrated in Fig. 3A. A polymer sample which was blended with VE prior to $\gamma$-irradiation shows low levels of crosslinking when compared to that of a similar sample but in which the hindered phenol antioxidant, VE, was infused in the polymer after the polymer was crosslinked (also with $\gamma$-irradiation) showing a much higher level of crosslinking (Fig. 3A); both methods of introducing vitamin $\mathrm{E}$ in the polymer are used commercially. Similar results were obtained with other hindered phenol AOs in the peroxide-initiated crosslinking process. Figure 3B illustrates this in PEXa pipes and shows clearly that the use of the conventional hindered phenol Irganox 1076 (Scheme 1, II) in PEXa pipe does interfere with the crosslinking process giving rise to a much lower level of polymer crosslinking compared to a similarly produced PEXa pipe but in the absence of the AO (Unst-PE). These problems would lead not only to premature failure of the products but also to associated health and safety concerns.

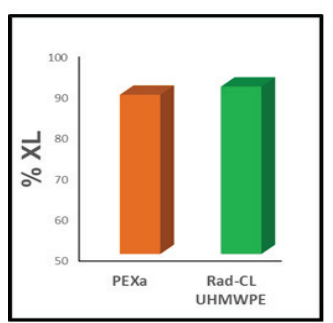

Fig 1. \% Crosslinking of a $\mathrm{PEX}_{\text {a }}$ pipe \& e-beam-irradiated (50 kGy dose) UHMWPE (both without AOs).

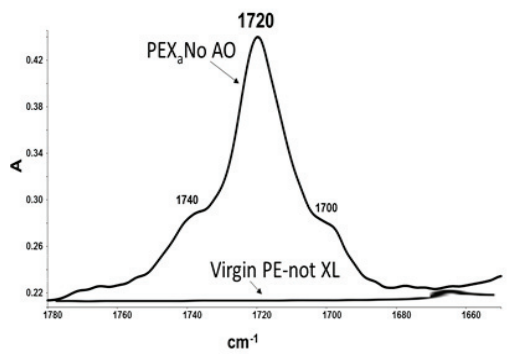

Fig 2. Oxidation of $P E X_{a}$ Engle Pipe (without AO).

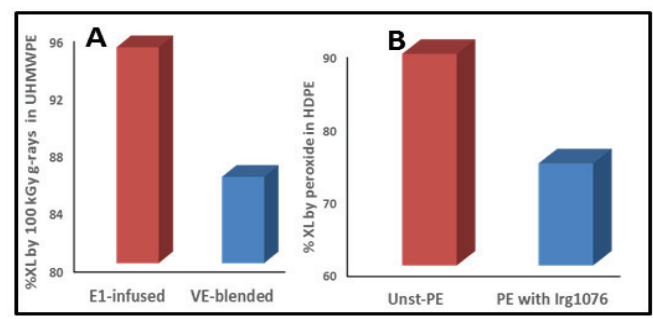

Fig 3. (A) $\gamma$-radiation crosslinked UHMWPE with infused VE (E1) and blended VE; (B) peroxide-crosslinked PEX $\mathrm{a}$ pipes (w/out 1076). 


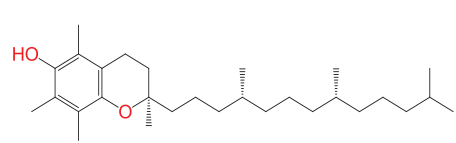

$\alpha$-tocopherol (vitamin E), I

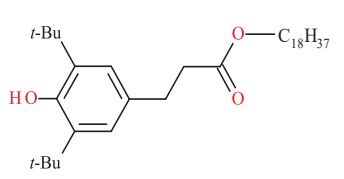

Irganox 1076, II

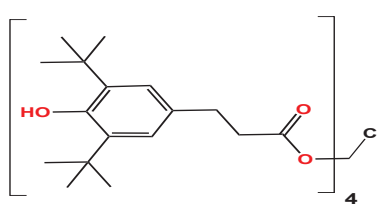

Irganox 1010, III

Scheme 1. Structures of antioxidants used.

\section{SAMPLE PREPARATION AND TESTING}

For preparation of orthopaedic implant samples, unstabilised medical-grade UHMWPE (GUR 1020) was used and prepared with different antioxidants (@ $0.6 \mathrm{w} / \mathrm{w} \%$ ) by an intense blending process. The polymer-AO mixture was then consolidated by compression moulding at $190^{\circ} \mathrm{C}$ under pressure to prepare thick bar stocks $(20-35 \mathrm{~mm})$ using either a standard laboratory moulding machine or a larger one which is actually used in the commercial manufacturing process. The consolidated polymer samples were then subjected to high energy irradiation using either $\gamma$-rays or electron beam (under inert atmosphere) at varying doses of 50-125 kGy (typical doses used commercially for crosslinking of UHMWPE with $\gamma$ - for knee implants is $\sim 90-100 \mathrm{kGy}$ and $\sim 25-35 \mathrm{kGy}$ for device sterilisation). A commercial sample of a tibia based on $\gamma$-irradiated UHMWPE containing VE infused after the crosslinking process (referred to here as E1) was also used. For testing, the tibia sample and the thick crosslinked stock bars were either microtomed to produce thin films $(50-150 \mu \mathrm{m})$ or cut to the required size and shape for mechanical testing.

For the preparation of peroxide crosslinked HDPE pipes $\left(\mathrm{PEX}_{\mathrm{a}}\right)$, unstabilised HDPE was used and formulated in the presence of a peroxide with a hindered phenol antioxidant, either Irganox 1076 or a synthesised AO in this work, using a commercial ram extrusion (Engel) manufacturing processes.

The extent of AO-retention in the crosslinked polymer samples was determined after purification of the polymer through a sequential solvent extraction protocol (dichloromethane followed by xylene) and the polymer was further characterised by spectroscopic methods. The overall extent of the polymer crosslinking was determined according to ASTM 2765-01 method using xylene extraction. The long-term thermal stability (LTTS) of the samples was determined by measuring the Oxidative Induction Time (OIT, by DSC). Fourier transform infrared scanning microscopy was performed on the microtomed films to assess the AO distribution profile across the sample using thickness-normalised absorption signature of the AOs. Mechanical properties for XL-UHMWPE were measured before and after a standardised accelerated ageing protocol involving exposure to $5 \mathrm{~atm}$. oxygen at $70^{\circ} \mathrm{C}$ for 14 days.

\section{EFFECT OF CONVENTIONAL AND NEW ANTIOXIDANTS ON THE LONG-TERM STABILITY AND PERFORMANCE OF XLPE}

\subsection{AOs performance in Crosslinked UHMWPE bearing Surfaces for Knee Implants}

The type of antioxidants used in such highly crosslinked polymers plays a critical role due to the various challenges they pose in such demanding applications including:

i. the different levels of their interference with the polymer crosslinking process,

ii. the extent of the uniformity of their distribution across the device, and,

iii. their retention in the polymer and loss through migration to the human-contact media, giving rise to both premature failure and safety concerns and compromising the quality of life of the patient.

The challenge of interference of vitamin $E$ with the crosslinking process of UHMWPE with increasing doses of $\gamma$ irradiation was examined and compared with that of a new AO-system (N-PPP). Figure 4 shows that increasing the irradiation dose increases the level of crosslinking in the polymer. However, at all irradiation doses examined, vitamin $\mathrm{E}$ (blended before $\gamma$-irradiation) is shown to give rise to a lower extent of crosslinking compared to the new AO, N-PPP; the level of crosslinking achieved $(>80 \%)$ by both AOs under the 'normal' crosslinking radiation dose of $100 \mathrm{kGy}$ is acceptable for this application. The lower overall crosslinking level shown by vitamin $\mathrm{E}$ is explained by the fact that it is known [5] to be one of the most effective free radical scavenging hindered phenols and it should therefore be expected to interfere with the radiation-initiated free-radical crosslinking process more than other antioxidant systems.

To examine the uniformity of distribution of both VE and the new AO, FTIR-microscopy was used to generate colour contour images of the stabilised XLPE polymer samples that represent the differences in the intensity of the main infrared fingerprint absorption indices (relative to polyethylene) for each AO. Figure $\mathbf{5}$ shows the contours of both the XLPE containing the infused VE (E1) and N-PPP. The new antioxidant N-PPP shows clearly a much more highly uniform distribution across the depth of the polymer sample compared to that of VE. 
As to the question of $\mathrm{AO}$ retention which is relevant to the safety aspects associated with their migration and release into the human-contact media, the advantage of the new AO system (N-PPP) is very clear since after an exhaustive solvent extraction it gives an almost complete retention $(>95 \%)$ in the highly crosslinked UHMWPE (using $100 \mathrm{kGy}$ dose of E-beam) which contrasts with the observed complete loss of VE, and that of another conventional hindered phenol, Irganox 1010, Scheme 1, III (the latter was used also and crosslinked sample produced in the same way), see Fig. 6A. The higher level of polymer crosslinking and excellent homogenous distribution of the new AO in the XLPE is reflected also in its excellent stabilising performance clearly seen from the much more effective long term oxidative stabilisation of the polymer (as measured from DSC-OIT) which is 5fold higher than that achieved by VE and that of Irg1010, see Fig. 6B. To further confirm the efficient stabilising performance of the new AO-system, the radiation-crosslinked UHMWPE samples were subjected to the ageing protocol described above (in section 3) followed by mechanical properties (UTS, EB \& impact strength) and oxidative stability (using carbonyl oxidation index) tests conducted before and after the accelerated ageing. The excellent performance of samples containing the new antioxidant N-PPP was clearly demonstrated by the comparable tensile properties and higher impact resistance (requiring higher energy-to-break) found before ageing compared to those of the commercial vitamin E-containing sample (E1). In addition, this XL-UHMWPE sample containing the AO N-PPP has also shown outstanding long-term thermal stability (oxidation index) that did not change after exposure to the accelerated ageing protocol.

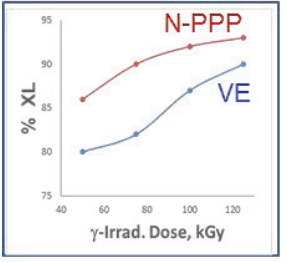

Fig.4. \% crosslinking vs radiation dose in UHMWPE.

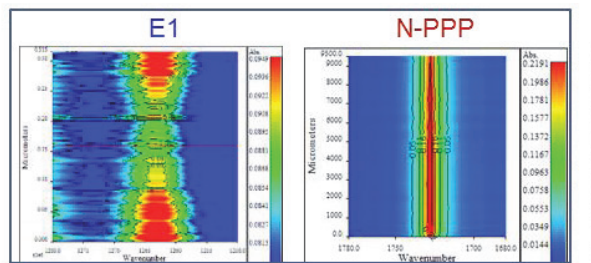

Fig 5. Mic-FTIR colour maps of radiation-XL-UHMWPE containing infused VE, E1, and a new AO, N-PPP.
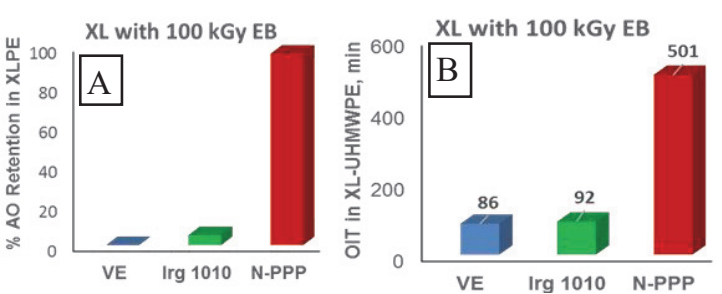

Fig. 6. (A) \% AO retention in XL-UHMWPE

(B) DSC-OIT for the same samples.

\subsection{Performance of AOs in Peroxide-Crosslinked HDPE for Potable Water Pipes, PEXa}

For peroxide-crosslinked HDPE water pipes, similar results were obtained for the level of interference of conventional hindered phenol antioxidants, such as Irganox 1076, with the crosslinking process of the PEX $\mathrm{X}_{\mathrm{a}}$ pipes. It was shown [4] that increasing the concentration of Irganox 1076 decreases the extent of the polymer crosslinking which confirms its interference with the peroxide-initiated free radical crosslinking process. Similarly, this hindered phenol was shown to have a low level of retention losing more than half its initial concentration after exhaustive solvent extraction. A different hindered phenol containing a reactive polymer function synthesised in this work was, on the other hand, shown to be retained to a very high extent in PEXa pipes that were produced in the same process.

In conclusion, conventional hindered phenol antioxidants, both natural and synthetic, interfere with the crosslinking process of PE polymers regardless of the method of crosslinking (peroxide-initiated or high energy radiation-initiated). The new AO-system used in XL-UHMWPE shows a much lower extent of interference with the radiation-crosslinking process. Further, the new AO system used in this medical application shows a dramatic increase in the level of its retention in the crosslinked polymer (after exhaustive solvent extraction) and this was paralleled by a very homogenous distribution across the thickness of the sample, with excellent oxidative stability (LTTS) and mechanical properties (before and after accelerated ageing) compared to VE, which is the approved antioxidant in current use for hip and knee prosthesis.

\section{REFERENCES}

1. O K Muratoglu, C R Bragdon, D O O'Connor, A S Morali Jasty, W H Harris, J Arthroplasty, 16, 149 (2001)

2. H A Khonakdar, J Morshedian, U Wagenknecht, S N Jafari, Polymer, 44, 4301 (2003)

3. E Oral, O K Muratoglu, Int. Orthopaedics, $\underline{\mathbf{3 5}}, 215$ (2011)

4. S. Al-Malaika, S. Riasat, C. Lewucha, Polym Degrad Stab, 145, 11 (2017)

5. G W Burton, K U Ingold, JACS, 104, 6472 (1981) 